\title{
NEUE BILDER FÜR ALTE RITUALE: DIE SATURN-STELEN ALS KULTMEDIEN IM RÖMISCHEN NORDAFRIKA
}

\author{
Günther Schörner
}

\section{Einführung*}

Ausgangspunkt der folgenden Überlegungen sind zwei Tatsachen. Erstens: Jeder oder annähernd jeder im Römischen Reich verehrte eine oder mehrere Gottheiten. Zweitens: Der wichtigste und signifikanteste Akt dieser Verehrung war das Opfer, speziell das Tieropfer. ${ }^{1}$ Deshalb wäre zu erwarten, dass Darstellungen dieses Rituals im ikonographischen Repertoire der römischen Kunst eine weite Verbreitung fanden. Die Verteilung von entsprechenden Kultdarstellungen ist jedoch in geographischer Hinsicht sehr unausgewogen: ${ }^{2}$ Während zahlreiche Opferdarstellungen aus West-Anatolien erhalten sind, sind nur ein paar wenige in Zentral- und Ost-Anatolien bezeugt. Monumente, die ein solches Ritual zeigen, sind in Gallien und Germanien stark vertreten, jedoch selten in Hispanien, und sie fehlen fast vollständig im römischen Griechenland. Die in dieser Hinsicht ergiebigs-

* Mein Dank gilt Prof. Dr. L. de Blois, Prof. Dr. A. Chaniotis, Prof. Dr. O. Hekster, Prof. Dr. E. Stavrianopoulou und Prof. Dr. C. Witschel für die Einladung zum Workshop des Internationalen Netzwerkes 'Impact of Empire' in Heidelberg. Für Diskussionsbeiträge und weitere Hinweise danke ich den Teilnehmern in Heidelberg, vor allem C. Auffarth, N. Belayche, A. Chaniotis und W. Eck. Eine frühere Version dieses Beitrags, jedoch mit einem anderen Schwerpunkt, wurde 2006 in Cambridge (UK) im Rahmen der 'Theoretical Roman Archaeology Conference' vorgetragen: G. Schörner, 'New images for old rituals: stelae of Saturn and personal cult in Roman North Africa', in: B. Croxford et al. (Hrsg.), TRAC 2006. Proceedings of the Sixteenth Theoretical Roman Archaeology Conference, Cambridge 2006 (Oxford 2007), 92-102.

${ }^{1}$ Zum Tieropfer: G. Wissowa, Religion und Kultus der Römer (München 1912, 2. Aufl.), 409-432; M. Beard - J. North - S. Price, Religions of Rome I: A History (Cambridge 1998), 36-37; J. Rüpke, Die Religion der Römer (München 2001), 136153; J. Scheid, An Introduction to Roman Religion (Edinburgh 2003), 79-106.

${ }_{2}$ Allgemein: I.S. Ryberg, Rites of the State Religion in Roman Art (Rome 1955); J. Ronke, Magistratische Repräsentation im römischen Relief. Studien zu standesund statusbezeichnenden Szenen (Oxford 1987); jetzt auch: V. Huet - J. Scheid, in: Thesaurus Cultus et Rituum Antiquorum I.(Los Angeles 2004), 183-235 (mit der weiteren Literatur). 
te Region ist freilich das römische Nordafrika, d.h. im Wesentlichen die Provinzen Africa proconsularis und Numidia.

Diese unausgewogene Verteilung von Opferdarstellungen sollte zur Vorsicht mahnen, alle bildlichen Wiedergaben in derselben Art und Weise zu interpretieren. Der erste Schluss, der aus dieser Beobachtung gezogen werden kann, ist, dass es zwar offenbar eine religiöse Notwendigkeit gab, den Göttern zu opfern, es aber gleichzeitig nicht erforderlich war, dies auch im Bild festzuhalten; zumindest waren Opferdarstellungen nicht in gleicher Weise unumgänglich wie Götterdarstellungen. In einem nächsten Schritt können wir folgern, dass es Gründe von regionaler Signifikanz für Ritualdarstellungen gegeben haben muss, weil ihre geographische Verteilung so ungleich ist. Diese Besonderheit, d.h. die Wirksamkeit kulturell begrenzter Spezifika innerhalb des großen, aus heterogenen Einzelkulturen zusammengesetzten römischen Reiches, kann besonders gut anhand von Opferdarstellungen aus den Provinzen Africa proconsularis und Numidia - dem modernen Tunesien und dem Ostteil Algeriens - diskutiert werden.

\section{Die Stelen im Saturnkult Nordafrikas}

Die überwiegende Anzahl von Opferdarstellungen aus Nordafrika befindet sich auf Stelen für Saturn, der allgemein als der romanisierte Nachfolger des punischen Ba al Hamon gilt. Die Stelen wurden in der großen Arbeit von Marcel LE GLAY über den „Saturne africain“ zu einem großen Teil erstmals zusammengestellt. ${ }^{3}$ Hauptziel dieser Untersuchung war es zu zeigen, wie sich der Kult Saturns unter der

${ }^{3}$ M. Le Glay, Saturne africain. Monuments I: L'Afrique proconsulaire (Paris 1961); id., Saturne africain. Monuments II: Numidie - Maurétanies (Paris 1966) (= Le Glay 1966a); M. Le Glay, 'Nouveaux documents, nouveaux points de vue sur Saturne africain', in: E. Lipiński (Hrsg.), Karthago. Acta Colloquii Bruxellensis 1986 (Leuven 1988), 187-237. Weitere Stelen finden sich in Einzelaufsätzen, zum Beispiel: A. Mahjoubi, 'Stèles à Saturne d'el-Afareg', Cahiers Tunesiennes 15 (1967), 147-156; A. Ben Younes, 'Stèles de Thibaris et de ses environs', Reppal 5 (1990), 27-42; M. Ghaki, 'Les stèles d'el-Ghzaizya', Reppal 7-8 (1992/93), 165177; Z. Benzina Ben Abdallah, 'Sur une collection d'antiques. Stèles à Saturne et epitaphes figurés', Africa 17 (1999), 11-24; zu einer der spätesten Stelen: A. Beschaouch, 'Une stèle consacrée à Saturne le 8 novembre 323', Bulletin Archéologique du Comité des Travaux Historiques et Scientifiques N.S. B 4 (1968), 253-268. 
Herrschaft Roms veränderte, also wie er ,romanisiert' wurde. ${ }^{4}$ Demonstriert wurde dies von LE GLAY anhand der Ikonographie sowohl des Saturn selbst als auch der zugehörigen Opferdarstellungen.

Die Stelen, die am stärksten am gewöhnlichen Erscheinungsbild römischer Reliefs orientiert sind und die deshalb auch immer wieder angeführt werden, sind dreigeteilt (Abb. 1): Im oberen Feld befinden sich Saturn und seine göttlichen Begleiter beziehungsweise die ihnen zugeordneten Symbole; es folgt die Darstellung des Opfernden im meist besonders großen Mittelfeld, während im kleineren unteren Bildfeld gewöhnlich das Opfertier entweder mit oder ohne weiteres Kultpersonal gezeigt wird. Insbesondere das mittlere Bildfeld erscheint vertraut, weil es am stärksten italischen Opferdarstellungen ähnelt: Der Opfernde, häufig mit Toga und Tunica bekleidet, steht an einem Altar und bringt Opfergut dar, indem er es in der Altarflamme verbrennt. ${ }^{5}$

Aufgrund dieser ikonographischen Übereinstimmungen nahm man an, dass sich italisch-stadtrömische und afrikanisch-provinziale Ikonographie wie auch die Formen der Kultausübung selbst immer mehr angenähert hätten und man in diesem Sinne von einer Ritualdynamik im römischen Nordafrika sprechen könne. Das grundlegende Modell hierbei ist das der ,Romanisierung', d.h. der bewussten und gleichmäßigen Ausbreitung römischer Elemente in allen Kulturbereichen, wobei Rom aufgrund seiner Machtposition zur treibenden Kraft geworden sei. Die bisher betrachteten Stelen bilden jedoch nur einen Ausschnitt aus dem Gesamtspektrum von Reliefs, die dadurch gekennzeichnet sind, dass sie einer Gottheit geweiht waren, die im Lateinischen als Saturnus bezeichnet wird. Die dreiteiligen Stelen mit reichem Dekor sind zwar besonders qualitätsvoll, stehen jedoch keineswegs allein und können deshalb nur im Kontext mit den anderen, weniger aufwändig verzierten Exemplaren sowie aus ihrer Funktion heraus interpretiert werden. Mit Blick auf die Gesamtheit der Saturnweihungen ist nämlich offensichtlich nicht die Opferdarstel-

\footnotetext{
${ }^{4}$ M. Le Glay, Saturne Africain. Histoire (Paris 1966) (= Le Glay 1966b) passim, bes. 243-244; E.R. Varner, 'Two portrait stelae and the romanization of North Africa', Yale University Art Gallery Bulletin (1990), 11-19; T. Kraus, 'Die Felsreliefs am Tempelberg', in: F. Rakob (Hrsg.), Simitthus I: Die Steinbrüche und die antike Stadt (Mainz 1993), 86.

${ }^{5}$ Zu Opferdarstellungen in Rom selbst vgl. vor allem Ryberg 1995, a.a.O. (Anm. 2); F. Fless, Opferdiener und Kultmusiker auf stadtrömischen historischen Reliefs. Untersuchungen zur Ikonographie, Funktion und Benennung (Mainz 1995).
} 
lung das Entscheidende, sondern ihre Eigenschaft als Stelen und deren Funktion innerhalb eines spezifischen Rituals.

Ausgehend von Untersuchungen in Saturnheiligtümern in Henchir el-Hami, ${ }^{6}$ Thuburnica (Sidi Ali bel Kassem), ${ }^{7}$ Siagu, ${ }^{8}$ Henchir Ghayadha ${ }^{9}$ oder Menzel Harb ${ }^{10}$ ist nämlich zu konstatieren, dass die Stelen in erster Linie dazu dienten, ein Opferdepot zu markieren (Abb. 2). Beim der Durchführung dieses Rituals wurden die Knochen des Opfertieres, das anscheinend als Ganzes verbrannt worden war, eingesammelt und in einer Urne im Bereich des eingezäunten, aber sonst kaum architektonisch gestalteten Heiligtums zusammen mit zerschlagenem Küchengeschirr beigesetzt. Zur Markierung dieses Opferdepots wurde dann zumeist eine Stele aufgestellt. ${ }^{11}$

Die Stelen dienten also vor allem zur Dokumentation der Tatsache, dass ein Opfer stattgefunden hatte. Ginge man nur von der Opferdarstellung aus, so würden grundsätzliche funktionale Divergenzen verwischt: Die Unterschiede zu den römischen Staatsreliefs mit Opferdarstellung oder auch zu Bildern von Ritualen auf Münzen oder Theaterfriesen in kleinasiatischen Städten sind aufgrund des vollkommen anderen funktionalen Kontextes einfach zu groß, wie stark auch die Ähnlichkeiten in der Darstellung sein mögen. Zu betonen sind dabei insbesondere zwei Punkte: Zum einen muss man sich vor Augen halten, dass die Stele kein autarkes Monument war, sondern auf mindestens zwei kultische Vorgänge verwies, nämlich die Gelobung eines Opfers und seine Einlösung nicht nur mittels der Aufstellung eines Steines, sondern auch durch den Vollzug des Opfers selbst. Zum zweiten ist aufgrund epigraphischer Quellen und der archäologischen Evidenz auf weitere kultspezifische Rituale an den Stelen zu schließen. So wurden diese anscheinend geschmückt, wie

${ }^{6}$ A. Ferjaoui, 'De Ba'al Hammon à Saturne. Présentation d'un sanctuaire à Henchir el-Hami (Tunisie centrale)', in: P. Donati Giacomini - M. L. Uberti (Hrsg.), Fra Cartagine e Roma. Seminario di Studi Italo-Tunisino, Bologna 2001 (Faenza 2002), 59-77.

${ }^{7}$ Le Glay 1961, a.a.O. (Anm. 3), 274-276.

${ }^{8}$ A. Merlin, Le sanctuaire de Baal et de Tanit prés de Siagu (Paris 1910).

${ }^{9}$ A. Ferjaoui - A. M'charek, 'Le sanctuaire de Ba'al Hammon-Saturne a Henchir Ghayadha', Reppal 5 (1990), 117-148.

${ }^{10}$ L. Foucher, 'Un sanctuaire néo-punique a Menzel Harb', Africa 1 (1966), 119129.

${ }^{11}$ Zum Beispiel in Henchir el-Hami als eine der neuesten Grabungen: Ferjaoui 2002, a.a.O. (Anm. 6), 65-69. 
man aus Durchbohrungen an vielen Stelen (Abb. 3) in unterschiedlichen Heiligtümern schließen kann. ${ }^{12}$

Außerdem wurden vor den Stelen weitere Opfergaben niedergelegt, bisweilen in speziell hierfür angefertigten ,Spendentafeln', also Steinplatten mit Eintiefungen für die Deponierung von Lebensmitteln. ${ }^{13}$ Schließlich ist epigraphisch noch die sogenannte dealbatio bezeugt, bei der es sich um das Streichen der Stelen mit weißer Farbe gehandelt haben dürfte. ${ }^{14}$ Die Stelen waren somit in ein komplexes Ritualgeschehen eingebunden, woran sich unzweifelhaft zeigt, dass nicht das Bild an sich, sondern der Bildträger die entscheidende GröBe darstellte. Deshalb ist auch Vorsicht geboten, wenn man - wie bisher oft geschehen - den gesamten Ritualkomplex ausgehend von einem eher sekundären Bestandteil erschließen möchte.

Dass die Saturn-Denkmäler nicht wie stadtrömische Monumente interpretiert werden dürfen, geht auch daraus hervor, dass ihre Dekoration sehr stark vom jeweiligen Aufstellungsort abhängig war. So ist die Gestaltung der Stelen innerhalb eines einzelnen Heiligtums in der Regel relativ homogen, unterscheidet sich aber von Heiligtum zu Heiligtum deutlich (Abb. 4a-b). ${ }^{15}$

Deshalb ist es durchaus möglich, allein an Größe, Umriss und natürlich an Umfang, Verteilung und Gestaltung des figürlichen und ornamentalen Schmucks auf ein bestimmtes Heiligtum bzw. eine bestimmte Region zu schließen. Natürlich kam es schon aufgrund des Vorhandenseins bestimmter Werkstätten zu einem gewissen Konformitätsdruck, aber es bleibt dennoch anzumerken, dass die Stelen nicht dazu verwendet wurden, sich innerhalb eines Heiligtums durch eine besondere Qualität in der Gestaltung repräsentativ vom Umfeld abzusetzen, und dass in der Tat unter den verschiedenen Heiligtümern die Spannbreite der möglichen Gestaltungen ungemein groß ist.

${ }^{12}$ Le Glay 1961, a.a.O. (Anm. 3), 225, 227 (ohne Interpretation beziehungsweise für Metallapplikationen in Anspruch genommen); zuletzt C. Mendleson, Catalogue of Punic Stelae in the British Museum (London 2003), 11.

${ }^{13}$ Le Glay 1966b, a.a.O. (Anm. 4), 305-306; als Beispiel vgl. auch Kraus 1993, a.a.O. (Anm. 4), 89.

${ }^{14}$ Marmortafel aus Henchir es-Srira mit Inschrift: Pro salute $p$ (atroni) $n$ (ostri) et Passi/enil[lae---] liberorum/que [---Ma]crin/us libe[rtus e?]or(um) deal/bavit petra[m S]aturni: Le Glay 1961, a.a.O. (Anm. 3), 310f. Nr. 3; allgemein: Le Glay 1966b, a.a.O. (Anm. 4), 349-350.

${ }^{15}$ Ein besonders gutes Beispiel stellen die weit über 300 Stelen von Thignica (Ain Tounga) dar: Le Glay 1961, a.a.O. (Anm. 3), 126-202. 
Die Frage nach der ,Romanisierung' des Bildschmuckes kann somit sowohl hinsichtlich der Methode als auch des Ergebnisses nicht den Kern der Sache treffen. Es ist deshalb auch nicht verwunderlich, dass der gewohnten und auch bei den Saturnstelen angewandten soziologischen Interpretation schnell Grenzen gesetzt sind: Während in Cuicul ein vilicus entsprechend der allgemeinen Charakteristik der dortigen Reliefs eine sehr gut gearbeitete, figurenreiche Stele aufstellte, ${ }^{16}$ ist aus der Großstadt Hippo Regius keine einzige ähnlich qualitätvolle Stele bekannt. ${ }^{17}$ Der gängige Konnex zwischen guter Qualität nach römischen Maßstäben und hoher sozialer Stellung kann deshalb nicht zutreffen, es sei denn, man ginge davon aus, dass es in Cuicul nur reiche und in Hippo nur arme Saturn-Anhänger gegeben hätte. Da den Stelen kein repräsentativer Charakter zu attestieren ist, ist es somit schwierig, von der Qualität der Reliefs auf die soziale Position zu schließen. ${ }^{18}$

\section{Kontinuität und Wandel}

Die Einbindung der Stelen in den Kult ist jedoch noch in anderer Hinsicht entscheidend. Ausschlaggebend für das Gesamtverständnis der Stelen ist, dass sich eine eindeutige Ritualkontinuität feststellen lässt. ${ }^{19}$ Sowohl in punischer als auch in neopunischer Zeit sind in Heiligtümern für Ba'al Hamon archäologische Überreste zu greifen, die auf eine über lange Zeit gleich bleibende Ritualabfolge schließen lassen. Auch die punischen und neopunischen Stelen dienten dazu, Urnen mit dem entsprechenden Opferbrand zu markieren. In einigen Heiligtümern wie in Thuburnuc oder bei den neuen Grabungen von Henchir el-Hami lässt sich eine kontinuierliche Nutzung und, soweit dies mit archäologischen Mitteln möglich ist, eine kontinuierliche

\footnotetext{
${ }^{16}$ Le Glay 1966a, a.a.O. (Anm. 3), 224 Nr. 26 Taf. 2.

${ }^{17}$ Le Glay 1961, a.a.O. (Anm. 3), 434-451 Taf. 17-18.

${ }^{18}$ Den Konnex betont und maßgeblich für die jüngere Forschung ist Le Glay 1966b, a.a.O. (Anm. 4), 402-406.

19 J.B. Rives, Religion and Authority in Roman Carthage from Augustus to Constantine (Oxford 1995), 142; als Beispiel s. Ferjaoui 2002, a.a.O. (Anm. 6), 7273; anders, da immer noch die Diskontinuität betonend: S. Saint-Amans, Topographie religieuse de Thugga (Dougga). Ville romaine d'Afrique proconsulaire (Tunisie) (Bordeaux 2004), 76.
} 
Durchführung des Opferrituals nachweisen. ${ }^{20}$ Diese Tradition blieb auch dann bestehen, als sich wichtige Änderungen im Umfeld ergaben: In beiden Fällen bestand das Heiligtum zunächst nur aus einer umgrenzten freien Fläche ohne weitere architektonische Gestaltung. Dies änderte sich erst im Laufe des späteren 2. oder frühen 3. Jhs. n. Chr., als aufwändigere Tempelbauten errichtet wurden. ${ }^{21}$ Auch wenn die neue Architektur vermutlich in irgendeiner Form in das Ritual eingebunden wurde, blieben die archäologisch nachweisbaren Grundbestandteile - Urnen mit Opferbrand, zerschlagenes Essgeschirr und die Markierung des Depots mit Stelen - unverändert. Die römerzeitlichen Reliefs waren also in ein weitestgehend nach traditionellen Mustern ausgeführtes Kultgeschehen eingebettet. Oder medienspezifisch ausgedrückt: Das Mediensystem ,Stele“ wurde als Aufzeichnungssystem in römischer Zeit beibehalten.

$\mathrm{Zu}$ fragen ist daher, was sich denn in römischer Zeit überhaupt gewandelt hat. Aus den lateinischen Inschriften kann geschlossen werden, dass sich zum größeren Teil die Sprache des Kultes - zumindest soweit sie auf den Stelen festgehalten wurde - geändert haben muss. Dessen ungeachtet sind aber auch im philologischen Bereich die Traditionslinien zum vorrömischen Kult deutlich: Eine Möglichkeit, auf frühere Kultformen zurückzugreifen, bestand anscheinend darin, punische Wörter in die lateinischen Votivinschriften zu integrieren. So gilt eine Weihung einem abbadir, einem heiligen Stein $;{ }^{22}$ oder es wird eine Stele als meten (Gabe) dargebracht. ${ }^{23}$ Auch die Bezeichnung baetylus für bet el weist in dieselbe Richtung, jedoch erfolgte in diesem Fall eine weitgehende Latinisierung. ${ }^{24}$ Neben

${ }^{20}$ Thuburnica: Le Glay 1961, a.a.O. (Anm. 3), 274-276; Henchir el-Hami: Ferjaoui 2002, a.a.O. (Anm. 6), 72-73.

${ }^{21}$ J. Eingartner, Templa cum porticibus. Ausstattung und Funktion italischer Tempelbezirke in Nordafrika und ihre Bedeutung für die römische Stadt der Kaiserzeit (Rahden 2005), 17-32.

${ }^{22}$ CIL VIII 21481 = ILS 4478 (Zucchabar): Abaddiri sa/ncto culto/res iuniores / suis $\operatorname{sum}(p)$ tis / aram constitu(erunt) / pro [---]; zum Steinkult allgemein vgl. S. Ribichini, Poenus advena. Gli dei fenici e l'interpretazione classica (Rom 1985), 115125.

${ }^{23}$ Stele aus Teboursouk: Die bonu/et felice/fausta/statui(t)/METEN: M. Fantar, 'Formules propitiatoires sur des stèles puniques et néopuniques', in: J. Quaegebeur (Hrsg.), Ritual and Sacrifice in the Ancient Near East. Proceedings of the International Conference Organized by the Katholieke Universiteit Leuven 1991 (Leuven 1993), 132.

${ }^{24}$ CIL VIII 23283 (Thala): Saturno Aug(usto) sacr(um) L(ucius) Pos[tumius] // [---]mus baetilum cum columna d(e) s(uo) fecit; vgl. C. Rossignoli, 'Persistenza del 
der Verwendung von punischen Votivtermini kam es auch vor, dass Rituale mit ihrem alten punischen Namen bezeichnet wurden. Ein berühmtes Beispiel hierfür sind Stelen aus Nicivibus, ${ }^{25}$ in denen ein Opfer als molchomor (für Punisch molk omor = „Opfer eines Lammes") benannt wird. ${ }^{26}$

Ein weitere Möglichkeit, ältere Termini zumindest mittelbar weiterzuverwenden, bestand in deren Übersetzung. Besonders wichtig dabei sind Umformungen einzelner Kultformeln, die belegen, dass das Ritual auch in seinen Einzelschritten fortgeführt wurde. Das ist etwa der Fall bei bestimmten punischen Segenswünschen (b ym n'm $w$ brk), die wörtlich übersetzt als die bonu felici; die bonum faustum felici oder etwas stärker verwandelt in der Form quod bonum et faustum feliciter sowie quod bonum faustum felix sit erscheinen. ${ }^{27}$ Die italisch-römische Herleitung dieser afrikanischen Formel, die man bisher angenommen hat, kann ausgeschlossen werden, weil inzwischen punische Inschriften die Formel für das 3. Jh. v. Chr. belegen und zudem ein Bindeglied in direkten lateinischen Übersetzungen greifbar ist. ${ }^{28}$

Neben der Sprache änderte sich in vielen Regionen auch die Art und Weise, wie die Stelen gestaltet wurden. Die Zahl der Denkmäler, auf denen Gott und Stifter dargestellt waren, nahm deutlich zu, doch stellte dies kein grundlegendes, allgemeines Phänomen dar, sondern war primär abhängig vom Ort, sekundär von der Zeitstellung. Wie im sprachlichen Bereich traten viele der von den punischen Stelen bekannten Motive noch in römischer Zeit auf. ${ }^{29}$ Für die Behandlung

culto betilico nell'Africa romana: un'iscrizione da Thala (Tunisia)', in: A. Mastino (Hrsg.), L'Africa romana IX (Sassari 1992), 73-96.

${ }^{25}$ So in AE 1931, 60. Zu den Stelen vgl. Le Glay 1966a, a.a.O. (Anm. 3), 68-75, Nr. 1-5 Taf. 31, 1-2.

${ }^{26}$ Zum Begriff und Ritual grundlegend: J. Guey, 'Ksiba et à propos de Ksiba. Civitas Popthensis - Moloch et Molchomor', Mélanges d'Ecole Française de Rome (1937), 67-107; J.G. Février, 'Molchomor', Revue de l'Histoire des Religions 143 (1953), 8-18; S. Ribichini, 'Sacrum magnum nocturnum. Note comparative nelle stele di N'Gaous', Aula Orientalis 17-18 (1999/2000), 353-362.

${ }^{27}$ Fantar 1993, a.a.O. (Anm. 23), 125-133; V. Ferron, 'La formule BYM N'M WBRK des stèles votives puniques ou néopuniques de l'Afrique du Nord', Bulletin CEDAC 13 (1993), 36-54.

${ }^{28}$ Ferron 1993, a.a.O. (Anm. 27), 40-41.

${ }^{29} \mathrm{Zu}$ figürlichen Motiven auf punischen Stelen: M. Hours Miédan, 'Les représentations figurées sur les stèles de Carthage', Cahiers de Byrsa 1 (1950), 15-160; C. Picard, 'Les représentations de sacrifice MOLK sur les ex-voto de Carthage I', Karthago. Revue d'Archéologie Africaine 17 (1975/76), 67-138; C. Picard, 'Les re- 
dieser Bildelemente kann eine Typologie erstellt werden, wobei grundsätzlich folgende Möglichkeiten des Umgangs mit punischen Bildmotiven in römischer Zeit denkbar sind:

- Die Beibehaltung der Motive an gleicher oder anderer Stelle auf der Stele.

- Eine Umformung: Beispielsweise die Mondsichel, die in römischer Zeit anders orientiert ist als in punischer Zeit; oder als Sonderfall der Umformung die Anthropomorphisierung des Tanitsymbols, ${ }^{30}$ zum Beispiel auf Stelen aus Nicivibus. ${ }^{31}$

- Die Einbindung von Objekten in einen narrativen Kontext, wenn beispielsweise einzelne Symbole nicht mehr für sich alleine stehen, sondern von Personen in den Händen gehalten werden (Abb. 5). ${ }^{32}$

- Das Ausblenden/Vergessen. ${ }^{33}$

Bei anderen Stelen lassen sich zudem stilistische Veränderungen feststellen. Beispielsweise wurden in punischer Zeit sehr häufige Gestaltungsmittel wie das versenkte Relief oder Einritzungen zugunsten des erhabenen Reliefs oder einer insgesamt plastischeren Gestaltung aufgegeben. Zusammen mit der Übernahme narrativer Elemente dienten diese gestalterischen Neuerungen vor allem einer detaillierteren und lebendigeren Darstellung der Rituale und ihrer Stifter; sie besaßen also durchaus auch inhaltliche Bedeutung.

Trotz der weitgehenden Kontinuität in der praktischen Kultausübung gibt es nämlich durchaus auch Anzeichen für konzeptionelle Änderungen, und zwar sowohl im Charakter als auch der Qualität des Kultes. Insbesondere kann eine zunehmende Personalisierung und Individualisierung der Kultanhänger bei der Ausübung der Op-

présentations de sacrifice MOLK sur les ex-voto de Carthage II', Karthago. Revue d'Archéologie Africaine 18 (1976), 5-16; Mendleson 2003, a.a.O. (Anm. 12), 7-11.

${ }^{30}$ Zum sogenannten Tanitsymbol besonders einleuchtend: S. Brown, Late Carthaginian Child Sacrifice and Sacrificial Monuments in their Mediterranean Context (Sheffield 1991), 123-131.

${ }^{31}$ Fantar 1993, a.a.O. (Anm. 23) Abb. 2 (ohne Bezug im Text); Mendleson 2003, a.a.O. (Anm. 12), 9 Abb. 6.

${ }^{32}$ Beispielsweise die sogenannte losange auf Stelen aus Aïn Nechma oder Hippo Regius: Le Glay 1961, a.a.O. (Anm. 3), Taf. 15, 1; 15, 3; 15, 6; 18,3; zu diesem Motiv zuletzt: P.F. Ruiu, 'Per una rilettura del motivo a losanga in ambito votivo fenicio-punico', in: Actas del IV Congreso Internacional de Estudios Fenicios y Púnicos, Cádiz 1995 (Cadiz 2000), 669-674.

${ }^{33}$ Dies gilt vor allem für das sogenannte Flaschensymbol. $\mathrm{Zu}$ diesem allgemein: Brown 1991, a.a.O. (Anm. 30), 138-141. 
ferrituale für Saturn nach der Eingliederung in den römischen Herrschaftsbereich festgestellt werden. Dabei sind folgende Gesichtspunkte von erheblicher Bedeutung: Zunächst wurde der Schutz der eigenen Familie, vor allem der eigenen Kinder, in den Inschriften als Begründung für die Aufstellung einer Stele und somit für die Durchführung der gesamten Ritualsequenz (bestehend aus Gelübde, Opfer und Votiv) intensiver herausgehoben als in punischer Zeit. Sodann ist festzustellen, dass nach der epigraphischen Evidenz das Gelübde aufgrund einer direkten persönlichen Kontaktaufnahme des Gottes mit dem Weihenden in Form eines Traumes erfolgte - Ausdrücke wie ex visu, visu monitus, somnio iussus begegnen in den Inschriften ziemlich häufig. ${ }^{34}$ Schließlich hat man mit Hilfe zooarchäologischer Untersuchungen in den großen punischen Heiligtümern feststellen können, dass die Urnen als Opferbrand vor allem die Knochen von neu geborenen bis vier Wochen alten Lämmern enthielten. ${ }^{35}$ Da der Geburtstermin bei Schafen zeitlich eingrenzbar ist, müssen alle diese Opfer im Frühjahr stattgefunden haben. An römerzeitlichen Urnen sind bisher noch keine derartigen Untersuchungen durchgeführt worden, es gibt aber eine ganze Reihe von Stelen, bei denen der Tag ihrer Errichtung bzw. der Durchführung des Opfers angegeben ist. ${ }^{36}$ Die Auflistung der Tage, für die die Durchführung des Rituals bezeugt ist, zeigt eine annähernd gleichmäßige Verteilung über das gesamte Jahr hinweg (Abb. 6). Ein offizielles oder auch nur halboffizielles Fest, bei dem viele Menschen gleichzeitig die Ritualhandlungen ausführten, fand also anscheinend in römischer Zeit nicht mehr statt.

Auf diese Tendenz zur Individualisierung, Personalisierung und Privatisierung nahm man auch bei der Gestaltung der Stelen Rücksicht. Es fällt auf, dass nach der Einbindung in das Imperium Romanum vor allem jene römischen Darstellungselemente übernommen wurden, durch welche die einzelne Person oder die eigene Familie im Vergleich zur punischen Zeit stärker betont wurden. So wurden die Stelen konsequenter in Zonen gegliedert und dadurch ihre archi-

${ }^{34}$ Le Glay 1966b, a.a.O. (Anm. 4), 341-342.

${ }^{35}$ F. Fedele - G.V. Foster, 'Tharros: Ovicaprini sacrificali e rituale del tofet', Rivista di Studi Fenici 16 (1988), 40-42; R. Docter et al., 'Interdisciplinary research on urns from the Carthaginian tophet and their contents', Palaeohistoria 43-44 (2001/02), 424.

${ }^{36}$ Zum Beispiel einige Stelen aus dem Heiligtum am Djebel Bou Kornein: Le Glay 1961, a.a.O. (Anm. 3), 36-73. 
tektonische Grundform besser greifbar. Aufgrund dieses prononcierteren Aufbaus ist eine deutlichere Trennung in göttliche und menschliche Sphäre möglich. Eine Hierarchisierung zwischen Gott und Mensch ist an sich nicht ungewöhnlich, erstaunlich ist aber, dass es nun vor allem Einzelpersonen oder Familiengruppen waren, die unter das den Göttern vorbehaltene Bildfeld traten; die maximale Personenzahl ist hierbei vier. Bei größeren Opfergruppen wurden die Assistenzfiguren samt Opfertier in ein drittes, noch tieferes Register versetzt. Dies könnte als Ausdruck für eine zunehmende Hierarchisierung der Gesellschaft gewertet werden, doch ist dabei zu bedenken, dass, wie bereits angemerkt, solche vielfigurigen Szenen grundsätzlich erst in römischer Zeit gebräuchlich waren. Wahrscheinlich sollte auf diese Weise jener Personenkreis besser herausgehoben werden, der für das Ritual verantwortlich war bzw. für den das Ritual durchgeführt wurde. Denn laut den punischen und lateinischen Inschriften war es der primäre Sinn des Opferrituals - und somit Hauptanliegen der Opfernden -, einen persönlichen Bezug zwischen Saturn und dem Stifter mit den Seinen, insbesondere den Kindern, herzustellen.

\section{Die Stelen als Kultmedien}

Die Stelen sind folglich nur als Kultmedien verständlich. Allein aus diesem Grund wurden sie aufgestellt und nur mit Hilfe dieses Ansatzes können wir sie in sinnvoller Weise beschreiben. In den Stelen ist allerdings nur die materielle Dimension zu greifen, zur weiteren Erklärung muss deshalb die soziale und mentale Dimension mitberücksichtigt werden, und zwar in Form des Saturnkults mit den entsprechenden Ritualen und den daran beteiligten Kultteilnehmern. ${ }^{37}$ Dies ist umso entscheidender, als sich die Stelen nicht nach den gewohnten, für Rom gültigen Kriterien beurteilen lassen, folgen sie doch anscheinend nicht einem der fundamentalen Gestaltungsprinzipien: Anders als die meisten öffentlich aufgestellten, aber privat finanzierten Bildwerke in Rom bzw. Italien, aber auch im griechischen Osten, dienen die Saturnstelen nicht der kompetitiven Repräsentation.

${ }^{37} \mathrm{Zu}$ den verschiedenen Dimensionen im Kommunikationsprozess und deren Bedeutung: A. Erll, Kollektives Gedächtnis und Erinnerungskulturen: Eine Einführung (Stuttgart - Weimar 2005), 101-105. 
Primär können die Saturnstelen als Externalisierungen des Rituals begriffen werden. Ihre Aufstellung liefert grundlegende Informationen über Durchführung und Empfänger des Opfers, in vielen Fällen auch über den Auftraggeber. Die Art und Weise, wie dies geschah, weist darauf hin, dass man an einer Individualisierung interessiert war: Man wählte eine einzelne Stele und trug sich nicht in eine Liste ein. Noch wichtiger ist freilich ein anderer Aspekt, der der Kommemoration. Der spezifische Materialwert der Stelen als reliefierte Steine zeigt, dass sie eindeutig für ein dauerhaftes Bestehen konzipiert waren. Die Stelen wurden deshalb schon bei ihrer Produktion auch als Gedächtnismedien funktionalisiert.

Für die Art der Rezeption der Stelen ist entscheidend, dass sie zusammen mit vielen anderen Exemplaren aufgestellt waren, die nicht nur ähnlich aussahen, sondern auch aus einem vergleichbaren Anlass errichtet worden waren. ${ }^{38}$ Stelen für Saturn mit Darstellungen von Opfern für Saturn wurden in Heiligtümern des Saturn nach einem Opfer für Saturn aufgestellt. Diese Aussage klingt banal, gewinnt aber an Interesse, wenn man sich überlegt, dass Opferdarstellungen in Rom gerade nicht an religiösen Gebäuden wie Tempeln angebracht wurden, sondern an Monumenten wie Triumph- und Ehrenbögen oder Reliefsäulen bzw. Sarkophagen oder Silberbechern, ganz zu schweigen von den zahlreichen Münztypen. Im Unterschied zu diesen nicht eindeutig kultisch konnotierten Bildträgern in Rom besaß jede Darstellung einer Saturnstele ein fest definiertes Bedeutungsfeld, das keinen interpretativen Spielraum zuließ. Der erklären-

${ }^{38}$ Zur Frage der Rezeption in der römischen Kunst vgl. allgemein T. Hölscher, Staatsdenkmal und Publikum. Vom Untergang der Republik bis zur Festigung des Kaisertums in Rom. (Konstanz 1984); J. Elsner, Art and the Roman Viewer (Cambridge 1995); P. Zanker, 'Search for the Roman Viewer', in: D. Buitron - Oliver (Hrsg.), The Interpretation of Architectural Sculpture in Greece and Rome. Proceedings of a Symposium, National Gallery of Art, Washington 1992 (Washington 1997), 179-191; M. Beard, 'The spectator and the column: reading and writing the language of gesture', in: J. Scheid - V. Huet (Hrsg.), La Colonne Aurélienne: autour de la Colonne Aurélienne. Geste et image sur la colonne de Marc Aurèle à Rome (Turnhout 2000), 265-279; P. Zanker, 'Bild-Räume und Betrachter im kaiserzeitlichen Rom', in: A. Borbein - T. Hölscher - P. Zanker (Hrsg.), Klassische Archäologie. Eine Einführung (Wiesbaden 2000), 205-226; J.R. Clarke, Art in the Lives of Ordinary Romans. Visual Representation and Non-Elite Viewers in Italy, 100 B.C. - A.D. 315 (Berkeley 2003), passim, bes. 35-41; grundlegend allgemein die Beiträge in: W. Kemp (Hrsg.), Der Betrachter ist im Bild. Kunstwissenschaft und Rezeptionsästhetik (Berlin 1992, 2. Aufl.); sowie zuletzt M.J. Mondzain, 'Was ist: Ein Bild sehen?', in: B. Hüppauf - C. Wulf (Hrsg.), Bild und Einbildungskraft (München 2006), 107-120. 
de Kontext war vielmehr eindeutig, da die Stelen bei der Ausführung ähnlicher Rituale betrachtet wurden. Diese Annahme ist umso wahrscheinlicher, als die meisten Saturnheiligtümer außerhalb der Städte lagen, ${ }^{39}$ also nicht von ,Flaneuren' oder zufälligen Passanten in großen Mengen besucht wurden. ${ }^{40}$ Kontext und Bezug der Stelen waren also solchermaßen beschaffen, dass eine missverständliche Rezeption nahezu ausgeschlossen war - selbst ein gerade nur irgendwie als vierbeiniges Wesen erkennbares Objekt wird nicht als Hund oder Bär verstanden worden sein, sondern als Schaf, das in engem Bezug zu einem Opfer stand. Eine technisch ausgefeilte Darstellung war deshalb für das Verständnis der Stelen nicht notwendig: Der Ort der Wahrnehmung, die dabei ausgeübten Tätigkeiten und die Vergesellschaftung mit vielen anderen Stelen ähnlichen Charakters boten genügend Anhaltspunkte für die Deutung der Bildinhalte. Da der Reliefschmuck der Stelen für deren Verwendung sekundär war und auch die kompetitive Repräsentation als Gestaltungsanreiz weitgehend entfiel, ist es verständlich, dass die meisten Stelen sehr einfache Dekorationen aufwiesen. Der Erkenntnisvorgang war um so eindeutiger, als Abbildungen von Saturn, wie sie auf den Stelen vorkamen, in anderen Kontexten keine Rolle spielten: So gibt es keine vergleichbaren Motive oder Szenen auf Mosaiken in Häusern, als Verzierung auf Reliefsigillaten oder auf Münzbildern, und nicht einmal als Reliefs an Tempeln. ${ }^{41}$ Eine Fehlinterpretation oder eine falsche Zuordnung des Bildes zu einem nicht-kultischen Kontext waren somit praktisch ausgeschlossen.

Die Uniformität der Stelen in einem bestimmten Heiligtum ist bisher immer negativ beurteilt worden, nämlich im Sinne einer fehlenden Innovationskraft beziehungsweise künstlerischer Unfähigkeit, doch kann und sollte sie durchaus positiv gesehen werden. Die Ähnlichkeit der Monumente, die noch dadurch gesteigert wurde, dass man als Besucher den Blick über einen Wald gleichartiger Stelen schweifen lassen konnte und so ihre Gleichförmigkeit schnell visuell

${ }^{39}$ Zur periurbanen Lage der Heiligtümer: C. Rossignoli, 'Templi periurbani di Africa Proconsolare e Numidia: alcuni esempi', in: A. Mastino - P. Ruggeri (Hrsg.), L'Africa Romana X (Sassari 1994), 559-595.

${ }^{40}$ Zum Begriff des 'Flaneurs' in der Klassischen Archäologie: D. Favro, The Urban Image of Rome (Cambridge 1996).

${ }^{41}$ So zeigt zum Beispiel das Mosaik mit Monatsdarstellungen aus Thysdrus überwiegend stadtrömische Feste als charakteristische Rituale: L. Foucher, 'Le calendrier de Thysdrus', Antiquités Africaines 36 (2000), 63-108. 
erfassbar war, war ein entscheidender Faktor bei der Herausbildung einer kollektiven Identität. ${ }^{42}$ Die Stifter der Stelen und die Opfernden müssen sich ihrer gemeinsamen Zugehörigkeit zu einer Gruppe bei jedem Besuch des Heiligtums bewusst gewesen sein. De facto wurde in den Stelen für Saturn ein Ereignis und ein Medium des individuellen Gedächtnisses kollektiviert. ${ }^{43}$ Die Besucher des Heiligtums bildeten dadurch eine Erinnerungsgemeinschaft. Nicht zu vernachlässigen ist natürlich der göttliche Rezipient: Die Stele sollte - auf ewig, oder zumindest zu Lebzeiten des Stifters und seiner Kinder - die Erfüllung des Votums und dessen korrekte Durchführung gegenüber Saturn dokumentieren.

\section{Vergleiche mit den Nordwestprovinzen}

Zur besseren Einordnung in den reichsweiten Kontext, wie es gerade im Rahmen der Tagungsreihe Impact of Empire sinnvoll erscheint, aber auch zur besseren Akzentuierung soll abschließend ein Versuch unternommen werden, die Saturnstelen nicht archäologischstilistisch, sondern funktional mit ähnlichen Phänomen in den Nordwestprovinzen zu vergleichen. Wie Monumente in einem Kult gestaltet wurden, bei dem die Aufstellung eines Denkmals und dessen figürlicher Schmuck einen wesentlichen Bestandteil des Rituals bildeten oder vielleicht sogar das gesamte Ritual ausmachten, kann an den Iuppiter-Gigantensäulen in den germanischen Provinzen exemplifiziert werden. ${ }^{44}$ Obwohl auch hier römische und indigene Elemente

${ }^{42}$ Zum Begriff der Identität in den Altertumswissenschaften vgl. beispielsweise E. Stephan, Honoratioren, Griechen, Polisbürger. Kollektive Identitäten innerhalb der Oberschicht des kaiserzeitlichen Kleinasien (Göttingen 2002) mit der älteren Literatur; grundlegend: J. Straub, 'Personale und kollektive Identität. Zur Analyse eines theoretischen Begriffs', in: A. Assmann - H. Friese (Hrsg.), Identitäten (Frankfurt a. M. 1998), 73-104; B. Giesen, Kollektive Identität. Die Intellektuellen und die Nation 2 (Frankfurt a. M. 1999), bes. 118-119; C. Emcke, Kollektive Identitäten. Sozialphilosophische Grundlagen (Frankfurt a. M. - New York 2000); kritisch: W. Niethammer, Kollektive Identität. Heimliche Quellen einer unheimlichen Konjunktur (Reinbek 2000).

${ }^{43}$ Zum Begriff: Erll 2005, a.a.O (Anm. 37), 15-16.

${ }^{44} \mathrm{Zu}$ den Iuppiter-Gigantensäulen allgemein: G. Bauchhenss - P. Noelke, Die Iupitersäulen in den germanischen Provinzen (Köln - Bonn 1981); G. Woolf, 'Representation as cult: the case of the Jupiter-columns', in: W. Spickermann (Hrsg.), Religion in den germanischen Provinzen Roms (Tübingen 2001), 117-134; W. Spi- 
nebeneinander standen, wir uns also wiederum im Bereich der romanisierten Religion befinden, und es sich um eine private Form der Kultausübung handelte, ist hier die ikonographische Geschlossenheit weit größer als bei den Saturnstelen der Africa proconsularis, die sich zumindest von Heiligtum zu Heiligtum und somit von Kleinregion zu Kleinregion deutlich unterscheiden. Zwar existieren keine zwei wirklich identische Iuppiter-Gigantensäulen, und man kann zwei differierende Gruppen in der Germania inferior und der Germania superior unterscheiden, doch in keinem Fall ergibt sich das unregelmäßige Gesamtbild der nordafrikanischen Saturnstelen. Der Bildschmuck war offensichtlich bei den Saturnstelen - auch in seiner aufwändigsten Form - nicht Kerngedanke, sondern von sekundärer Bedeutung.

Viel engere Parallelen zum Saturnkult und seiner Votivpraxis lassen sich in einigen Heiligtümern in der Provinz Germania inferior wie dem Heiligtum von Empel an der Maas oder von Kessel, wo eine Kultkontinuität von der vorrömischen Eisenzeit bis in die Kaiserzeit festgestellt werden konnte. ${ }^{45}$ Trotz sich ändernder architektonischer Ausstattung blieb hier - wie in Nordafrika - die rituelle Praxis anscheinend unverändert bestehen. ${ }^{46}$ Ähnlich dem Saturnkult wurde auch die Votivgattung - im Falle der niederländischen Heiligtümer wurden vor allem Metallgegenstände wie Fibeln, Schwerter oder Geschirrteile sowie Münzen deponiert - beibehalten, obwohl sich in deren Gestaltung durch die Eingliederung in das Römische Reich Veränderungen ergeben hatten. ${ }^{47}$

ckermann, Germania Superior. Religionsgeschichte des römischen Germanien I (Tübingen 2003), 246-247.

${ }^{45}$ Grundlegend: T. Derks, Gods, Temples and Ritual Practices. The Transformation of Religious Ideas and Values in Roman Gaul (Amsterdam 1998); N. Roymans, Ethnic Identity and Imperial Power. The Batavians in the Early Roman Empire (Amsterdam 2004); zusammenfassend: P.S. Wells, Die Barbaren sprechen. Kelten, Germanen und das römische Europa (Darmstadt 2007; englische Originalausgabe Princeton 1999), 177-178.

${ }^{46}$ Derks 1998, a.a.O. (Anm. 45), 112-113, 177; Roymans 2007, a.a.O. (Anm. 45), 12-14, 129-131.

${ }^{47}$ Für eine andere Region wurde dies untersucht von I. Wellington, 'Considering continuity of deposition on votive sites in northeastern France from $200 \mathrm{BC}$ to AD 100', in: M. Carruthers et al. (Hrsg.), TRAC 2001. Proceedings of the Eleventh Annual Theoretical Roman Archaeology Conference Glasgow 2001 (Oxford 2002), 112. 


\section{Schluss}

Die Saturnstelen in Nordafrika sind sicherlich ein Beispiel, wie stark eine bestimmte Monumentengruppe - Reliefstelen - an einen bestimmten Ritualkomplex - Opferpraxis im Saturnkult - gebunden ist. Besonders deutlich wird dies in einem Vergleich mit Opferdarstellungen aus Kleinasien, wo wir ganz andere Medien als Bildträger vorfinden und Rituale vor allem auf den von den Städten geprägten Münzen bildlich gefasst sind. Auch ergeben sich große Unterschiede zur stadtrömischen Praxis der Platzierung und Verwendung von Kultdarstellungen. ${ }^{48}$ Die hier vorgelegte Fallstudie sollte deutlich gemacht haben, dass nur eine anwendungsorientierte, d.h. eine medienorientierte Betrachtung von Kultdarstellungen sinnvoll ist, und dass eine solche Betrachtung auch die besten Voraussetzungen bietet, den Veränderungen der Rituale und ihrer Medien gerecht zu werden.

Jena, Dezember 2007

${ }^{48}$ Vgl. hierzu jetzt auch G. Schörner, 'Opferdarstellungen im römischen Kleinasien: Kultikonographie zwischen Adaption und Distinktion - Rituale und Ikonographie in der Africa Proconsularis: Religiöses Handeln im Spannungsfeld von Eigenständigkeit und Integration: Eine Gegenüberstellung', in: J. Rüpke (Hrsg.), Antike Religionsgeschichte in räumlicher Perspektive (Tübingen 2007), 108-116. 


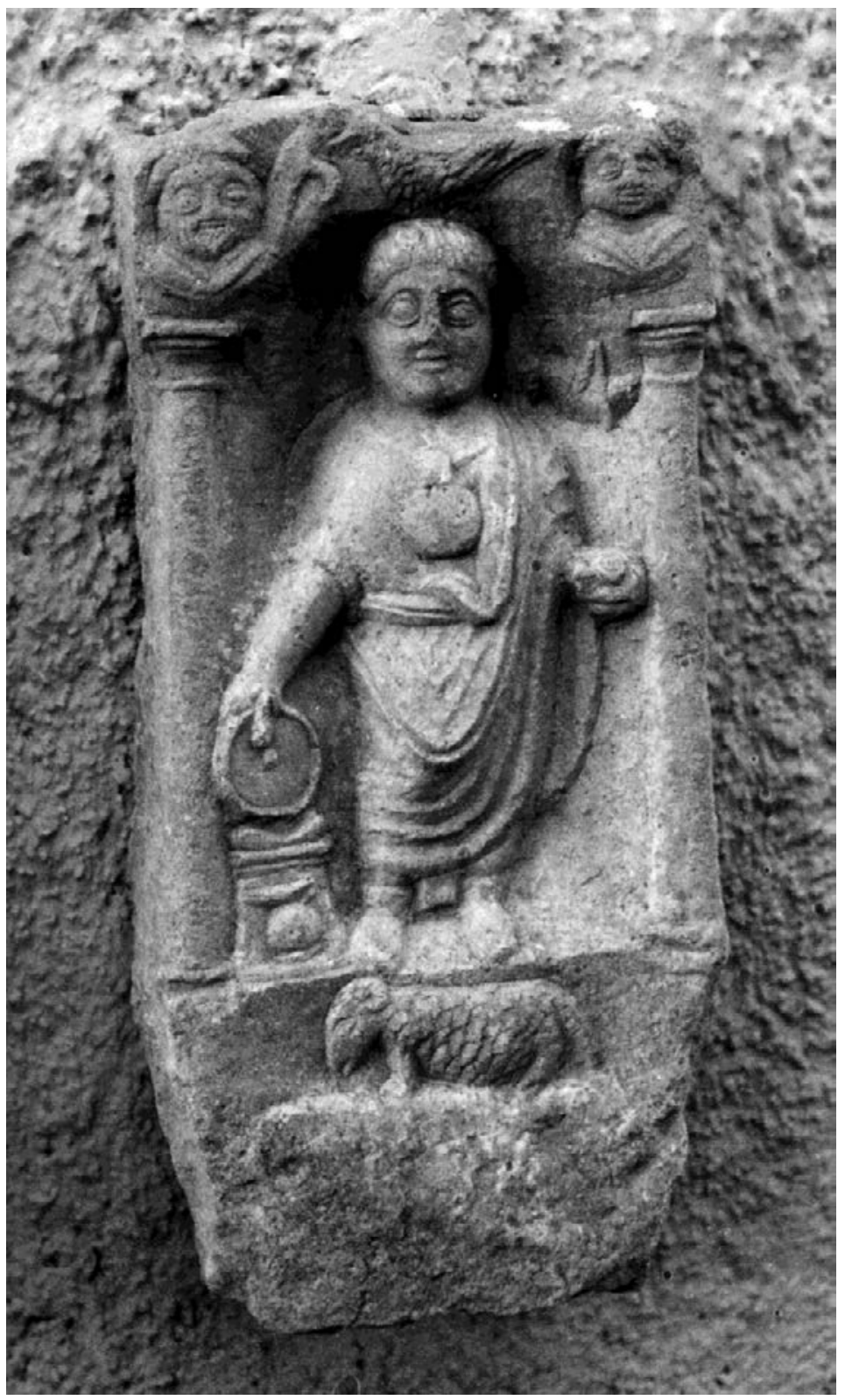

Abb. 1: Stele aus Timgad, Museum Timgad (Foto: Schörner, wie Le Glay 1966, a.a.O. (Anm. 3), Taf. 27,9) 


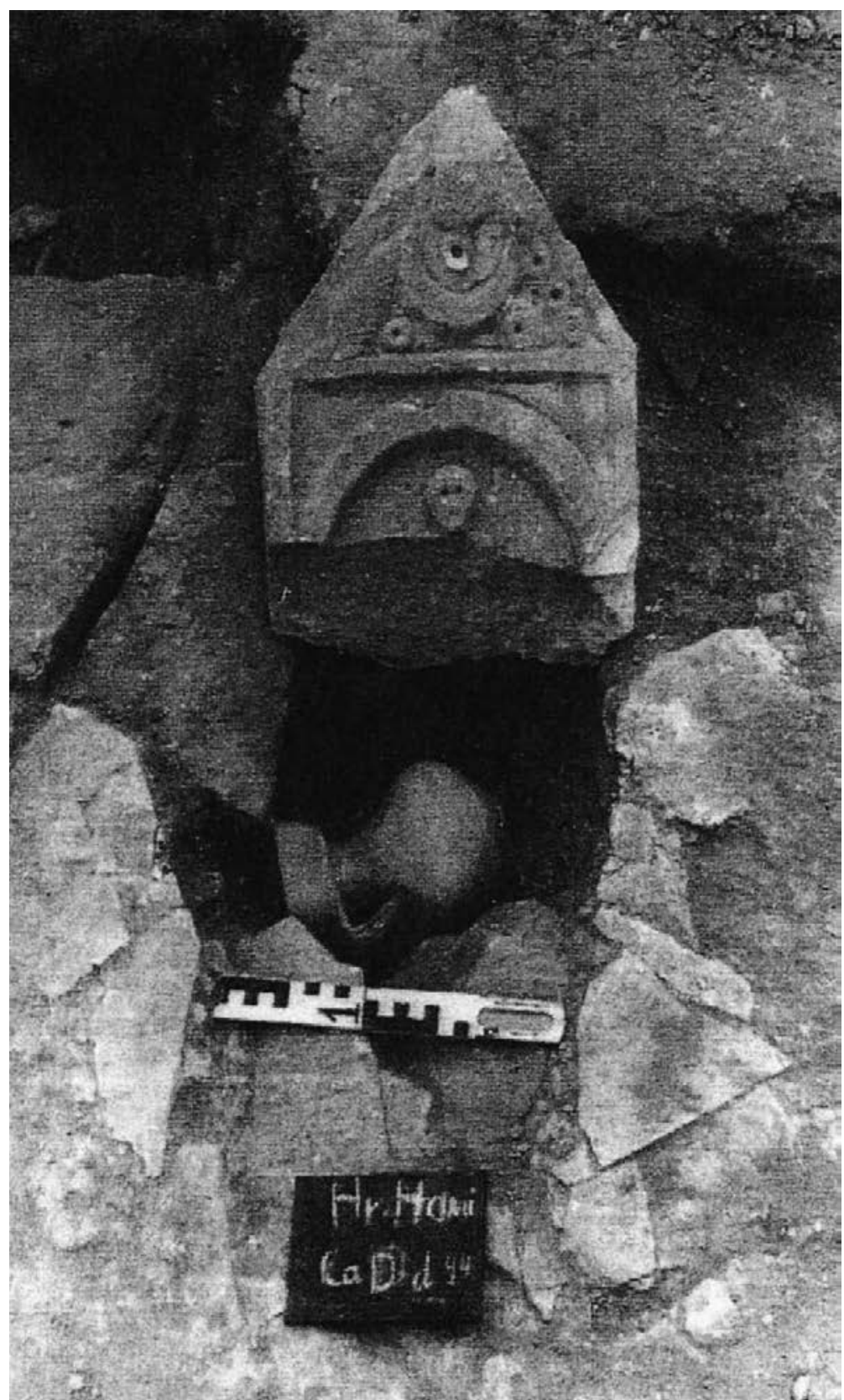

Abb. 2: Opferdepot mit Stele in Henchir el Hami

(nach Ferjaoui 2002, a.a.O. [Anm. 7], Abb. 1). 


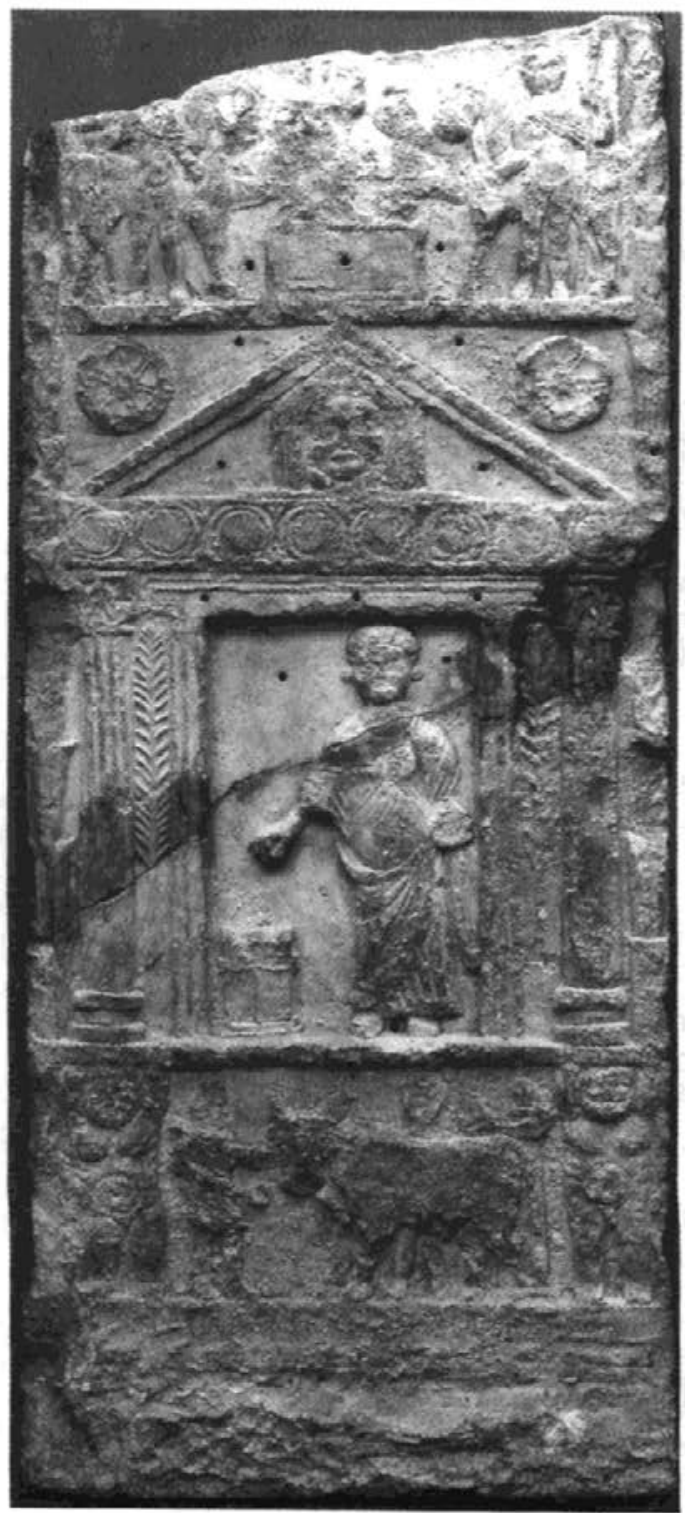

Abb. 3: Stele mit Bohrungen aus Zentraltunesien, British Museum Inv. NPu 45.125066 (nach Mendleson 2003, a.a.O. [Anm. 13], Abb. NPu 45). 


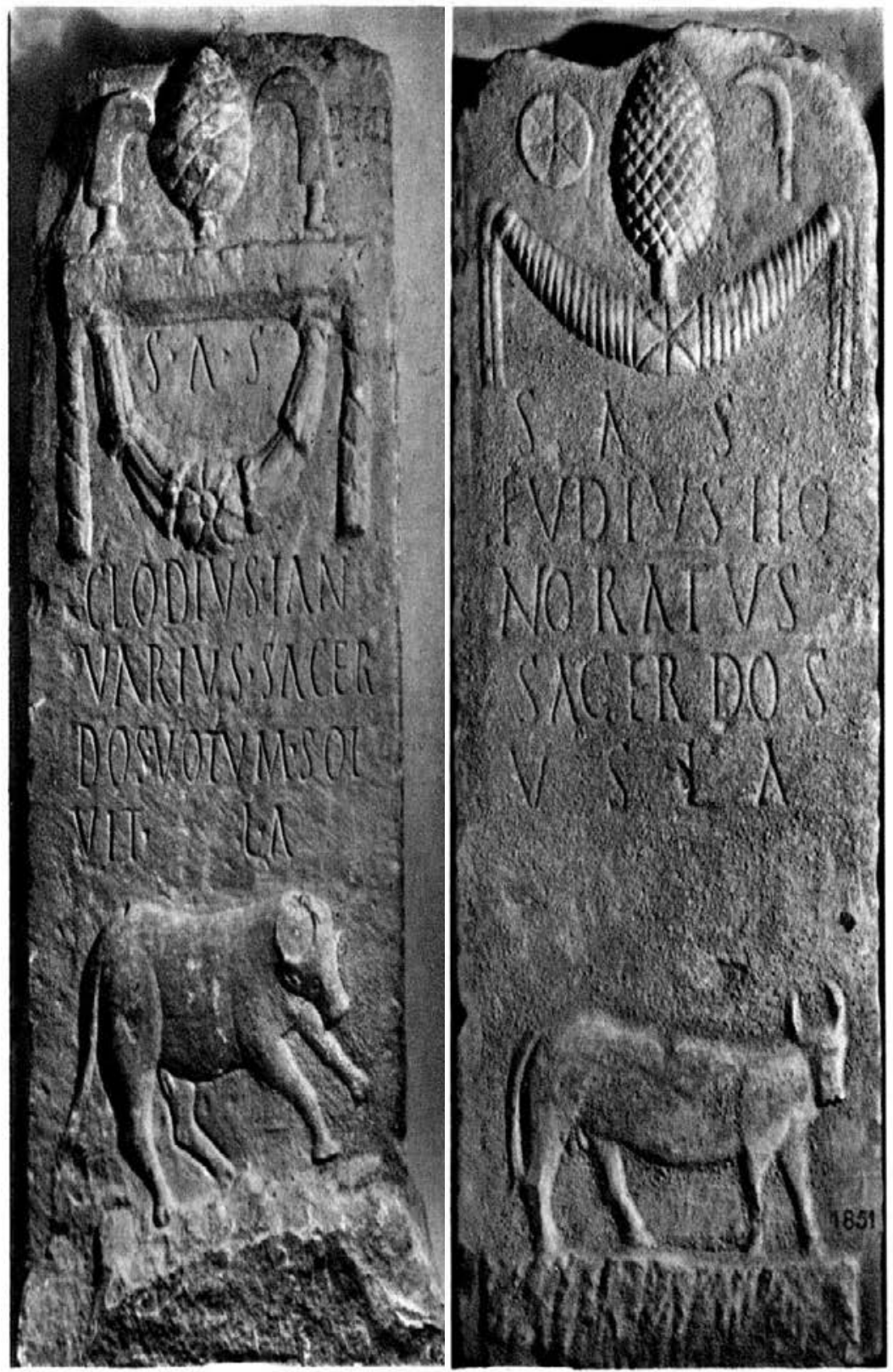

Abb. 4: Stelen aus Ain Tounga (nach Le Glay 1961, a.a.O. [Anm. 3], Taf. 5,3; 5,5). 


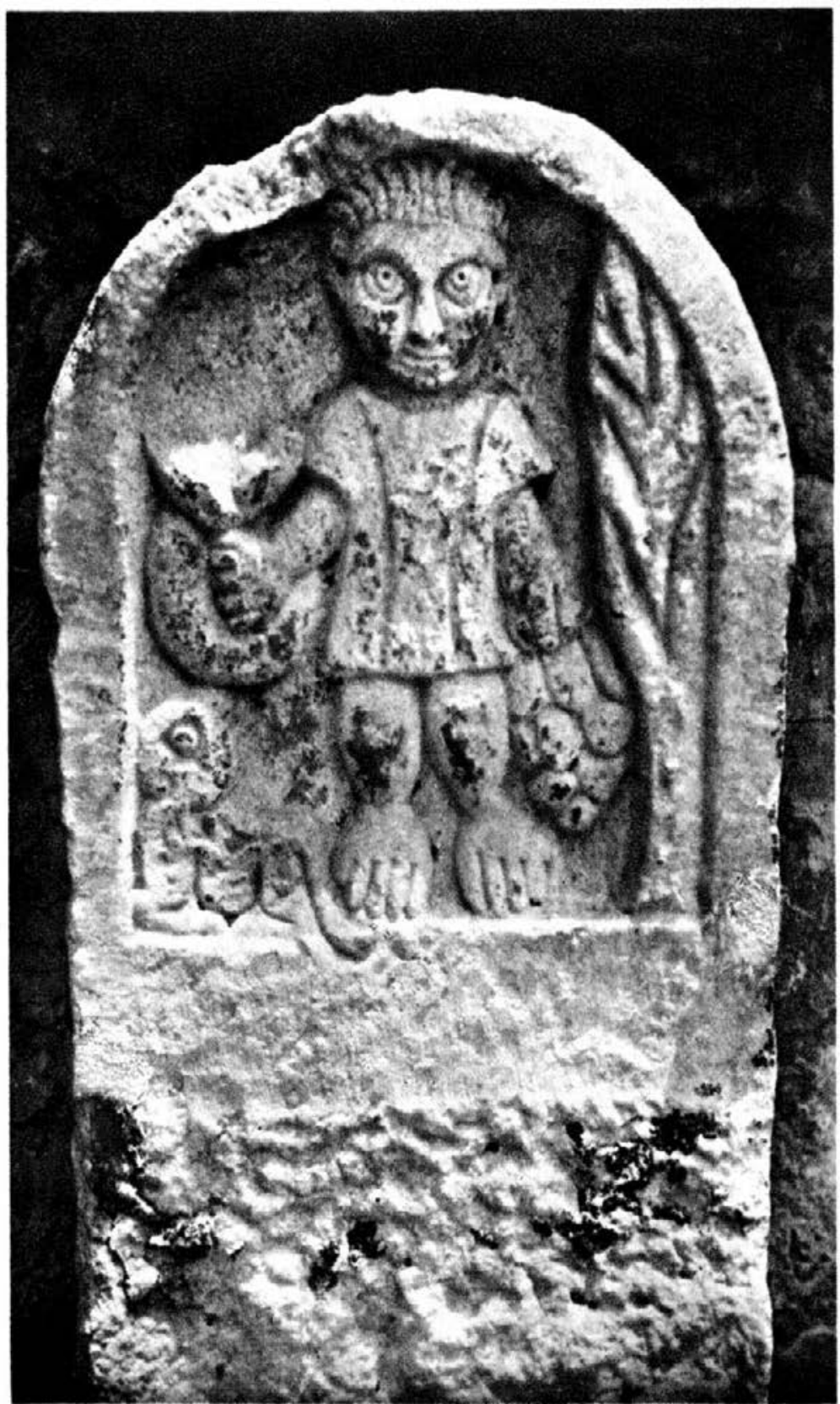

Abb. 5: Stele aus Hippo regius (nach Le Glay 1961, a.a.O. [Anm. 3], Taf. 18,4). 


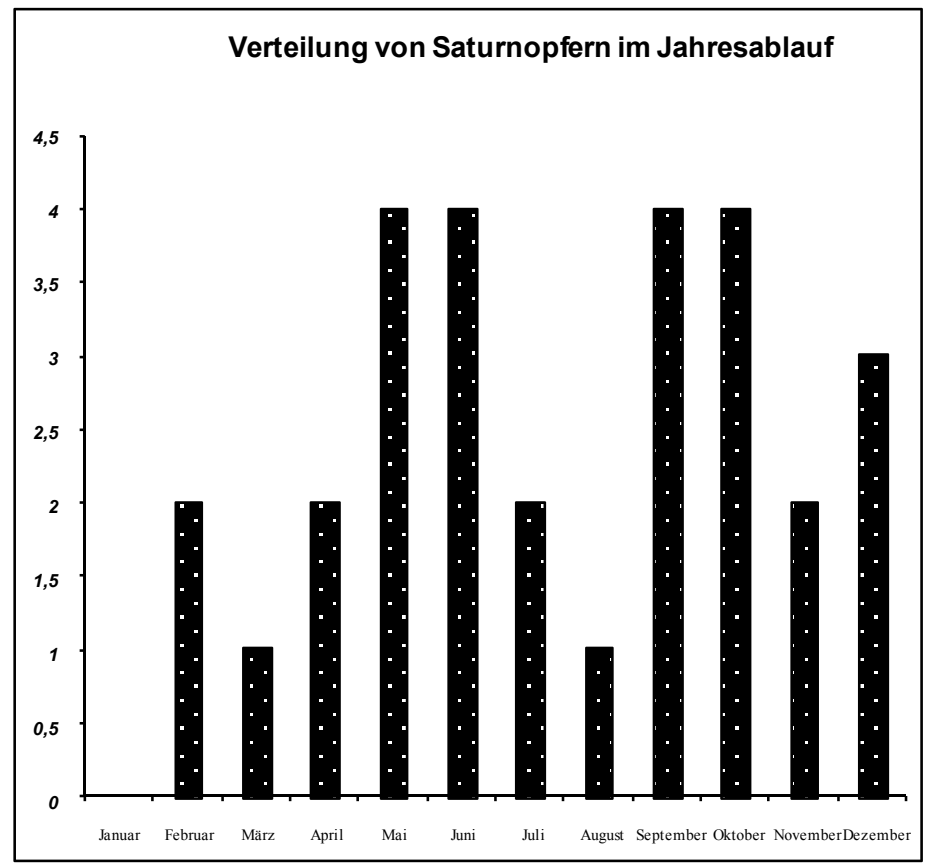

Abb. 6: Epigraphisch bezeugte Verteilung der Saturnopfer im Jahresablauf (Diagramm: Schörner). 